\title{
ON AN ESTIMATE FOR THE MODULUS OF CONTINUITY OF A NONLINEAR INVERSE PROBLEM ${ }^{1}$
}

\author{
Elena V. Tabarintseva \\ Computational Mathematics Department, South Ural State University, \\ Chelyabinsk, Russia, \\ eltab@rambler.ru
}

\begin{abstract}
A reverse time problem is considered for a semi-linear parabolic equation. Two-sided estimates are obtained for the norms of values of a nonlinear operator in terms of the norms of values of the corresponding linear operator. As a consequence, two-sided estimates are established for the modulus of continuity of a semi-linear inverse problem in terms of the modulus of continuity of the corresponding linear problem.
\end{abstract}

Key words: Parabolic equation, Inverse problem, Modulus of continuity of the inverse operator, Approximate method, Error estimate.

\section{Introduction}

The article examines the reverse time problem for a semilinear parabolic equation. V. K. Ivanov, V. N. Strakhov, and their disciples and followers developed the theory and worked out the technique to obtain error estimates for approximate methods of solution of linear ill-posed problems on compact sets (correctness classes) (see, for example, $[2,3,6]$ ). This theory naturally introduces the concepts of optimal and order-optimal approximate methods of solution of unstable problems. The relevant concepts were introduced for nonlinear ill-posed problems as well (see, for example, $[9,10])$ Various methods for solving nonlinear ill-posed problems were considered, for example, in $[1,5,7,8,11]$.

For linear ill-posed problems the technique for computing the error of optimal regularization method on a correctness class is based on the connection between the error of the method and modulus of continuity for the inverse operator, which can be calculated for each operator and each correctness class $M$ by means of the spectral technique [3,6]. For nonlinear problems, the connection between the error of the method and the modulus of continuity for the inverse operator is also present; unfortunately, there seems to be no known method for calculation of the modulus of continuity on correctness classes.

To the best of our knowledge, this paper is the first one to use the Volterra property of the operator corresponding to the reverse time problem to obtain two-sided estimates for the norms of values of a non-linear operator in terms of the norms of the values of the corresponding linear operator. This allows us to get two-sided estimates for the modulus of continuity for the semi-linear inverse problem on correctness classes through the modulus of continuity for the corresponding linear problem, for which the calculation technique is well-known.

${ }^{1}$ Published in Russian in Trudy Inst. Mat. i Mekh. UrO RAN, 2013. Vol. 19. No 1. P. 253-257. 


\section{An estimate of the modulus of continuity for the semi-linear inverse problem}

\section{1. "Forward" problem for a parabolic equation}

Consider an initial boundary value problem for a parabolic equation. That is, the function $v(x, t) \in C\left(\left[t_{0} ; T\right] ; W_{2}^{2,0}[0 ; l]\right) \cap C^{1}\left(\left(t_{0} ; T\right) ; L_{2}[0 ; l]\right)$ is to be determined from the following equations:

$$
\begin{gathered}
\frac{\partial v}{\partial t}=\frac{\partial^{2} v}{\partial x^{2}}+a(x) v+f(t, v(x, t)) ; \quad t \in\left(t_{0} ; T\right), \quad x \in(0 ; l), \\
v\left(t_{0}, x\right)=\varphi(x) \quad(0<x<l), \\
v(t, 0)=v(t, l)=0 \quad\left(0<t_{0}<t<T\right),
\end{gathered}
$$

where $a(x) \in C^{2}[0 ; l], \varphi(x) \in L_{2}[0 ; l]$ are certain given functions. Here $f:\left[t_{0} ; T\right] \times L_{2}[0 ; l] \rightarrow L_{2}[0 ; l]$ is a mapping that is Lipshitz continuous in $v$ and the Holder continuous in $t$ :

$$
\left\|f\left(v_{1}, t_{1}\right)-f\left(v_{2}, t_{2}\right)\right\|_{L_{2}[0 ; l]} \leq L\left\|v_{1}-v_{2}\right\|_{L_{2}[0 ; l]}+K\left|t_{1}-t_{2}\right|^{\alpha}
$$

for all $t_{1}, t_{2} \in\left[t_{0} ; T\right], v_{1}, v_{2} \in L_{2}[0 ; l]$, where the constants $L, K$ do not depend on $t, 0<\alpha<1$.

Let $X_{n}(x)$ denote the eigenfunctions of the Sturm-Liouville problem

$$
X_{n}^{\prime \prime}+a(x) X(x)=\mu X, \quad X(0)=X(l)=0
$$

corresponding to the eigenvalues $\mu_{n}=-\lambda_{n}^{2}$ and forming a complete orthonormal system in $L_{2}[0 ; l]$. Problem (1.1) is equivalent to the integral equation

$$
v(x, t)=\sum_{n=1}^{\infty} e^{-\lambda_{n}^{2} t} \varphi_{n} X_{n}(x)+\int_{0}^{t}\left(\sum_{n=1}^{\infty} e^{-\lambda_{n}^{2}(t-\tau)} f_{n}(\tau, v(x, \tau)) X_{n}(x)\right) d \tau,
$$

where $\left.f_{n}(t, v(x, t))=\int_{0}^{l} f(t, v(x, t)) X_{n}(x) d x ; \varphi_{n}(t, v(x, t))=\int_{0}^{l} \varphi(x)\right) X_{n}(x) d x$ (see, for example, $[4])$.

Consider the initial-boundary value problem for the linear parabolic equation corresponding to problem (1.1). Namely, the function $u(x, t) \in C\left(\left[t_{0} ; T\right] ; W_{2}^{2,0}[0 ; l]\right) \cap C^{1}\left(\left(t_{0} ; T\right) ; L_{2}[0 ; l]\right)$ is to be determined from the following equations:

$$
\begin{gathered}
\frac{\partial u}{\partial t}=\frac{\partial^{2} u}{\partial x^{2}}+a(x) u ; \quad t \in\left(t_{0} ; T\right), \quad x \in(0 ; l), \\
u\left(t_{0}, x\right)=\varphi(x) \quad(0<x<l) \\
u(t, 0)=u(t, l)=0 \quad\left(0<t_{0}<t<T\right) .
\end{gathered}
$$

Problem (1.3) has a unique solution, which can be represented in the form

$$
u(x, t)=\sum_{n=1}^{\infty} e^{-\lambda_{n}^{2} t} \varphi_{n} X_{n}(x)
$$

Here, $\varphi_{n}=\int_{0}^{l} \varphi(x) X_{n}(x) d x$ are the Fourier coefficients of $\varphi(x)$ with respect to the orthonormal system of functions $X_{n}(x)$ (see, for example, [4]). 
Lemma. Consider functions $\varphi_{1}, \varphi_{2} \in L_{2}[0 ; l]$. Let $u_{1}(x, t), u_{2}(x, t)$ be the corresponding solutions to the problem (1.3), let $v_{1}(x, t), v_{2}(x, t)$ be the solutions to the problem (1.1). Then, for every $t \in\left[t_{0} ; T\right]$, the following inequalities hold

$$
e^{-L T e^{L T}}\left\|u_{1}-u_{2}\right\| \leq\left\|v_{1}-v_{2}\right\| \leq e^{L T}\left\|u_{1}-u_{2}\right\|
$$

P r o o f. It follows from equalities (1.2) and (1.4) that

$$
\begin{gathered}
v_{1}(x, t)-v_{2}(x, t)=u_{1}(x, t)-u_{2}(x, t) \\
+\int_{0}^{t}\left(\sum_{n=1}^{\infty} e^{-\lambda_{n}^{2}(t-\tau)}\left(f_{n}\left(\tau, v_{1}(x, \tau)\right)-f_{n}\left(\tau, v_{2}(x, \tau)\right) X_{n}(x)\right)\right) d \tau .
\end{gathered}
$$

Thus, taking into account the Lipshitz continuity of $f$, we obtain the inequality

$$
\left\|v_{1}(x, t)-v_{2}(x, t)\right\| \leq\left\|u_{1}(x, t)-u_{2}(x, t)\right\|+L \int_{t_{0}}^{t}\left\|v_{1}(x, \tau)-v_{2}(x, \tau)\right\| d \tau .
$$

The estimate below follows from (1.6) by the Gronwall lemma:

$$
\left\|v_{1}(x, t)-v_{2}(x, t)\right\| \leq e^{L T}\left\|u_{1}(x, t)-u_{2}(x, t)\right\| .
$$

From equality (1.5), we can also obtain the following:

$$
\begin{gathered}
u_{1}(x, t)-u_{2}(x, t)=-\left(v_{1}(x, t)-v_{2}(x, t)\right) \\
+\int_{0}^{t}\left(\sum_{n=1}^{\infty} e^{-\lambda_{n}^{2}(t-\tau)}\left(f_{n}\left(\tau, v_{1}(x, \tau)\right)-f_{n}\left(\tau, v_{2}(x, \tau)\right) X_{n}(x)\right)\right) d \tau
\end{gathered}
$$

hence, taking into account the Lipschitz continuity, we get

$$
\left\|u_{1}(x, t)-u_{2}(x, t)\right\| \leq\left\|v_{1}(x, t)-v_{2}(x, t)\right\|+L \int_{t_{0}}^{t}\left\|v_{1}(x, \tau)-v_{2}(x, \tau)\right\| d \tau .
$$

Moreover, in view of (1.7), inequality (1.8) implies that

$$
\left\|u_{1}(x, t)-u_{2}(x, t)\right\| \leq\left\|v_{1}(x, t)-v_{2}(x, t)\right\|+L e^{L T} \int_{t_{0}}^{t}\left\|u_{1}(x, \tau)-u_{2}(x, \tau)\right\| d \tau .
$$

From (1.9), by the Gronwall lemma, we have

$$
\left\|u_{1}(x, t)-u_{2}(x, t)\right\| \leq e^{L T e^{L T}}\left\|v_{1}(x, t)-v_{2}(x, t)\right\| .
$$

The statement of lemma follows from inequalities (1.7) and (1.10). 


\subsection{The inverse problem for a parabolic equation}

Consider the reverse time problem for a semi-linear parabolic equation. That is, we have to determine a function $\varphi(x) \in L_{2}[0 ; l]$ such that the solution of initial-boundary value problem (1.1) satisfies the condition

$$
v(x, T)=\chi(x),
$$

where $\chi(x) \in L_{2}[0 ; l]$ is a given function from the range of the forward problem. Namely, we assume there exists a function $\varphi(x) \in L_{2}[0 ; l]$ such that the forward problem takes it to $\chi(x)$, where $\chi(x)$ is given explicitly.

Simultaneously, we consider the inverse problem for the corresponding linear equation. Let $\hat{\chi}(x)$ denote the solution to linear forward problem (1.3) with the initial condition $u(0, x)=\varphi(x)$, $0<x<l$, and consider the inverse problem with the following condition:

$$
u(x, T)=\hat{\chi}(x),
$$

where $u(x, t)$ is the solution of initial boundary value problem (1.3) for the linear equation. Hence, in parallel with nonlinear inverse problem (1.1), (1.11), we consider the inverse problem for the linear equation, i. e., we have to determine a function $\varphi(x) \in L_{2}[0 ; l]$ such that the solution to initial boundary value problem (1.3) satisfies condition (1.12).

Let $M \subset L_{2}[0 ; l]$ be a compact set. We assume that, for a given function $\chi(x) \in L_{2}[0 ; l]$, nonlinear inverse problem (1.1), (1.11) has an exact solution $\varphi(x)$ belonging to the set $M$, but the values of the function $\chi(x)$ are unknown; instead we know approximate values of the given function, that is, we know a function $\chi_{\delta} \in L_{2}[0 ; l]$ such that $\left\|\chi-\chi_{\delta}\right\|<\delta$. Given the initial data, we are to determine an approximate solution $\varphi_{\delta}$ to the reverse time problem and to estimate its deviation from the exact solution.

Consider the following values:

$\omega(M, \delta)=\sup \left\{\left\|\varphi_{1}-\varphi_{2}\right\|: \varphi_{1}, \varphi_{2} \in M,\left\|\chi_{1}-\chi_{2}\right\| \leq \delta\right\}$ is a modulus of continuity for the nonlinear inverse problem,

$\hat{\omega}(M, \delta)=\sup \left\{\left\|\varphi_{1}-\varphi_{2}\right\|: \varphi_{1}, \varphi_{2} \in M,\left\|\hat{\chi}_{1}-\hat{\chi}_{2}\right\| \leq \delta\right\}$ is a modulus of continuity for the linear inverse problem.

The following theorem holds.

Theorem. There exists $\delta_{0}>0$, such that for all $0<\delta<\delta_{0}$ the following inequalities hold:

$$
\hat{\omega}\left(M, e^{-L T} \delta\right) \leq \omega(M, \delta) \leq \hat{\omega}\left(M, e^{L T e^{L T}} \delta\right) .
$$

$\mathrm{P}$ r o o f. Consider $\varphi_{1}, \varphi_{2} \in M$. We estimate the value $\omega(M, \delta)$ using the inequalities obtained in the lemma.

Find the upper estimate of $\omega(M, \delta)$. Write inequality (1.10) for $t=T$ :

$$
\left\|\hat{\chi}_{1}-\hat{\chi}_{2}\right\| \leq e^{L T e^{L T}}\left\|\chi_{1}-\chi_{2}\right\| \text {. }
$$

Therefore, the conditions $\varphi_{1}, \varphi_{2} \in M,\left\|\chi_{1}-\chi_{2}\right\| \leq \delta$ implies that $\left\|\hat{\chi}_{1}-\hat{\chi}_{2}\right\| \leq \delta_{1}$, where $\delta_{1}=e^{L T e^{L T}} \delta$.

Thus, by definition of the modulus of continuity

$$
\omega(M, \delta) \leq \hat{\omega}\left(M, e^{L T e^{L T}} \delta\right) .
$$

Find the lower estimate of $\omega(M, \delta)$. Write inequality (1.7) for $t=T$ :

$$
\left\|\chi_{1}-\chi_{2}\right\| \leq e^{L T}\left\|\hat{\chi}_{1}-\hat{\chi}_{2}\right\| .
$$

Denote $\delta_{2}=e^{-L T} \delta$. Taking into account the inequality above, we see that the conditions $\varphi_{1}, \varphi_{2} \in$ $M\left\|\hat{\chi}_{1}-\hat{\chi}_{2}\right\| \leq \delta_{2}$ imply that $\left\|\chi_{1}-\chi_{2}\right\| \leq \delta$. Hence, by definition of the modulus of continuity,

$$
\omega(M, \delta) \geq \hat{\omega}\left(M, e^{-L T} \delta\right) .
$$

This completes the proof. 


\section{Examples}

Example 1. Consider the set $M_{1}$ of functions $\varphi(x) \in L_{2}[0 ; l]$ such that

$$
\begin{gathered}
\frac{\partial^{2 k} \varphi}{\partial x^{2 k}} \in L_{2}[0 ; l] \quad(k=1, \ldots, m), \\
\varphi(0)=\varphi(l)=0, \quad \varphi^{(2 k)}(0)=\varphi^{(2 k)}(l)=0 \quad(k=1, \ldots, m-1) ; \\
\left\|\frac{\partial^{2 m} \varphi}{\partial x^{2 m}}\right\|_{L_{2}[0 ; l]} \leq r .
\end{gathered}
$$

Calculating the modulus of continuity for problem $(1.3),(1.12)$ in the way suggested in $[1,11]$, we obtain

$$
\hat{\omega}\left(M_{1}, \delta\right)=2 r \frac{\left(T-t_{0}\right)^{m}}{\ln ^{m}(r / \delta)} .
$$

Using the theorem, we get the following estimate of the modulus of continuity for semi-linear problem (1.1), (1.11) on the set $M_{1}$ :

$$
\frac{2 r\left(T-t_{0}\right)^{m}}{\left(\ln \frac{r e^{L T}}{\delta}\right)^{m}} \leq \omega\left(\delta, M_{1}\right) \leq \frac{2 r\left(T-t_{0}\right)^{m}}{\left(\ln \frac{r}{L e^{L T} \delta}\right)^{m}} .
$$

Example 2. Consider $0<t_{0}<T$. We define the set $M_{2}$ using the linear problem

$$
\begin{gathered}
\frac{\partial u}{\partial t}=\frac{\partial^{2} u}{\partial x^{2}}+a(x) u ; \quad t \in(0 ; T), \quad x \in(0 ; l), \\
u(0, x)=u_{0}(x) \quad(0<x<l) \\
u(t, 0)=u(t, l)=0 \quad\left(t_{0}<t<T\right) .
\end{gathered}
$$

Denote $\varphi(x)=u\left(t_{0}, x\right)$. We consider the set of functions

$$
M_{2}=\left\{\varphi(x) \in L_{2}[0 ; l]:\left\|u_{0}\right\| \leq r\right\} .
$$

Find the estimate of the modulus of continuity for semi-linear inverse problem (1.1), (1.11) on the set $M_{2}$. Examine linear problem (1.3), (1.12). The theorem implies that

$$
\hat{\omega}\left(M_{2}, e^{-L T} \delta\right) \leq \omega\left(M_{2}, \delta\right) \leq \hat{\omega}\left(M_{2}, e^{L T e^{L T}} \delta\right) .
$$

The standard calculations for the linear problem (see, for example, [2]) allow us to obtain the estimate

$$
2 e^{-L t_{0} e^{L t_{0}}} r^{\frac{T-t_{0}}{T}} \delta^{\frac{t_{0}}{T}} \leq \hat{\omega}\left(M_{2}, \delta\right) \leq 2 e^{L t_{0}} r^{\frac{T-t_{0}}{T}} \delta^{\frac{t_{0}}{T}} .
$$

Finally, we obtain

$$
2 e^{L t_{0}\left(1-e^{L t_{0}}\right)} r^{\frac{T-t_{0}}{T}} \delta^{\frac{t_{0}}{T}} \leq \omega\left(\delta, M_{2}\right) \leq 2 e^{L t_{0}\left(1+e^{L T}\right)} r^{\frac{T-t_{0}}{T}} \delta^{\frac{t_{0}}{T}} .
$$

\section{REFERENCES}

1. Vasin V.V., Ageev A.L. Inverse and Ill-posed problems with a priori information. Inverse and IllPosed Problems Series. Utrecht: VSP, 1995. 255 p.

2. Ivanov V.K., Vasin V.V., Tanana V.P. Theory of linear ill-posed problems and its applications. Inverse and Ill-Posed Problems Series. Walter de Gruyter, 2002. 294 p.

3. Ivanov V.K., Korolyuk T.I. Error estimates for solutions of incorrectly posed linear problems //USSR Computational Mathematics and Mathematical Physics. 1969. Vol. 9, no. 1. P. 35-49.

4. Mikhlin S.G. Mathematical physics; an advanced course. Amsterdam, Norhth-Holland Pub.Co., 1970. $562 \mathrm{p}$. 
5. Bakushinsky A.B., Kokurin M.Yu. Iterative methods for approximate solution of inverse problems. Mathematics and its Applications. Vol. 577. Dordrecht: Springer, 2004. 291 p.

6. Strakhov V.N. On solving linear ill-posed problems in a Hilbert space // Diff. equations. 1970. Vol. 6, iss. 8. P. 1990-1995.

7. Tabarintseva E.V. On error estimation for the quasi-inversion method for solving a semi-linear illposed problem // Sib. Zh. Vychisl. Mat. 2005. Vol. 8, iss. 3, P. 259-271.

8. Tanana V.P., Tabarintseva E.V. On a method to approximate discontinuous solutions of nonlinear inverse problems // Sib. Zh. Vychisl. Mat. 2007. Vol. 10, iss. 2. P. 221-228.

9. Tanana V.P. On the convergence of regularized solutions of nonlinear operator equations // Sib. Zh. Ind. Mat. 2003. Vol. 6, iss. 3. P. 119-133.

10. Tanana V.P. Optimal order methods of solving non-linear ill-posed problems // USSR Computational Mathematics and Mathematical Physics, 1976. Vol. 16, iss. 2. P. 219-225.

11. Tikhonov A.N., Leonov A.S., Yagola A.G. Nonlinear ill-posed problems. Chapman \& Hall, 1998. $387 \mathrm{p}$. 